\title{
CMB Polarization Results from the QUIET Experiment
}

\section{Masaya Hasegawa for the QUIET Collaboration}

High Energy Accelerator Research Organization, KEK

E-mail: Masaya.Hasegawa@kek.jp

The Q/U Imaging ExperimenT (QUIET) is a ground-based CMB Polarization experiment, whose aim is to detect the degree-scale B-modes (curl components) induced by primordial gravitational waves. The existence of the primordial gravitational wave is a generic prediction of inflation, and hence the detection of the B-modes is a "smoking-gun" signature of the inflationary universe. The B-mode signal is expected to be orders of magnitude smaller than the temperature anisotropy so a large detector array with precise control and mitigation of systematic effects is essential. QUIET employs the world largest coherent receiver arrays in $43 \mathrm{GHz}$ and $95 \mathrm{GHz}$ frequency bands, and had accumulated CMB data for over 8000 hours at 5,000m altitude in Chile: the Chajnantor plateau. Our unique instruments design and the calibration strategy lead to the lowest levels of systematic errors to date. Those advantages enable us to begin to prove the B-modes. In this proceedings, we review the QUIET experiment and report the latest results for the CMB polarization measurements.

36th International Conference on High Energy Physics,

July 4-11, 2012

Melbourne, Australia 


\section{Introduction}

The faint pattern of cosmic microwave background $(\mathrm{CMB})$ polarization promises to provide new and interesting information on the universe. The primary goal of CMB polarization studies in the next decade is to detect the degree-scale B-modes (curl components) induced by primordial gravitational waves. The existence of the primordial gravitational waves is a generic prediction of inflation. Therefore the detection of the B-modes is a "smoking-gun" signature of inflation [1]. Furthermore, the energy scale of inflationary potential can be reconstructed from the power of the B-modes. Therefore, the CMB polarization experiment is quite attractive for high energy physicists, too.

The $\mathrm{Q} / \mathrm{U}$ Imaging ExperimenT (QUIET) is a ground-based program to measure the CMB polarization, and among the experiments aiming for the detection of the inflationary B-modes. The scientific observation was started with the $43 \mathrm{GHz}$ detector in October 2008. After nine months of observation, we deployed $95 \mathrm{GHz}$ detector, replacing $43 \mathrm{GHz}$ detector, and observed between August 2009 and December 2010. The first science result with $43 \mathrm{GHz}$ data have been published in [2], and here we mainly describe on the measurement of CMB polarization power spectra with the $95 \mathrm{GHz}$ data, as well as the instruments and observation.

\section{Instrument}

The QUIET telescope consists of a $1.4 \mathrm{~m}$ side-fed classical Dragonian antenna, which provides the angular resolution of $27 \mathrm{arcmin}$. (12 arcmin.) at $43 \mathrm{GHz}(95 \mathrm{GHz})$ frequency band. The optics is designed to probe degree scale angular scale at which the inflationary B-mode signature peaks. The telescope also has a co-moving ground-shield to suppress sidelobe response. The detector and telescope sit atop a 3-axis (azimuth, elevation, and "deck") mount system. By regularly rotating the third axis (deck), we modulated the instrumental-polarization axis, suppressing instrumentalpolarization systematic errors.

The detectors which form the focal planes use a highly compact design based on High Electron Mobility Transistors (HEMTs) that provides simultaneous measurements of the Stokes parameters Q, U, and I in a single detector [3]. In the first observing season, we deployed 17-elements detector array, with a central frequency of $43 \mathrm{GHz}$; in the second season we observed with 84-elements array at a central frequency of $95 \mathrm{GHz}$. The sensitivity of each array is $69 \mu K s^{1 / 2}$ and $87 \mu K s^{1 / 2}$, which were the best sensitivity among on-going CMB polarization experiments when we deployed.

The responses of each detector to the polarized signal are calibrated and monitored with regular calibration observation of astronomical and artificial sources. The detector angles (i.e., the orientations of the polarization responses) are calibrated to $0.5 \mathrm{deg}$. precision with the combination of Tau-A observations for absolute-angle determination and a sparse-wire-grid calibrator [4] for relative-angle determination over the focal plane. Sky dip (using the varying atmospheric thickness as a source) allows us to measure the spurious polarization of each detector with respect to the total power (so-called I-to-Q/U leakage) with $0.3 \%$ precision per calibration. The detector responsivities are calibrated using Tau-A and sky-dip data. This calibration strategies and the dedicated study lead to the lowest level of systematic errors as will be discussed in this paper. 


\section{Observation}

The observation site was the Chajnantor Test Facility on the Chajnantor Plateau in the Atacama Desert, Chile. The site is known as one of the best place over the world for measuring CMB because of its high altitude $(5080 \mathrm{~m})$ and low atmospheric water vapor. Under the median conditions, the zenith sky brightness at $95 \mathrm{GHz}(43 \mathrm{GHz})$ is about $5 \mathrm{~K}(10 \mathrm{~K})$.

We started scientific observations in October 2008 with the $43 \mathrm{GHz}$ (Q-band) receiver array. The observing season finished in June 2009, collecting 2668 hours of CMB data. Then we switched the instruments into the $95 \mathrm{GHz}$ (W-band) receiver array, and observed between August 2009 and December 2010, collecting 5337 hours of CMB data.

We observed four patches as shown in Fig. 11 each covering $15^{\circ} \times 15^{\circ}$ sky region. We selected the patches for their low synchrotron emission as measured by WMAP, and because the patches are separated at least $30^{\circ}$ from the Sun and Moon throughout the year, which allows uninterrupted observations throughout the season. Each patch is scanned with a periodical azimuth motion at fixed elevation (CES) for typically $\sim 1.5$ hours until it drifts out of the line of the sight. The elevation and azimuth are then changed to recenter the patch, and this process continues until the patch sets and another patch is selected. By scanning at constant elevation for each patch scan, we observe thought a constant column density of atmosphere and ensure a stable atmospheric contribution.

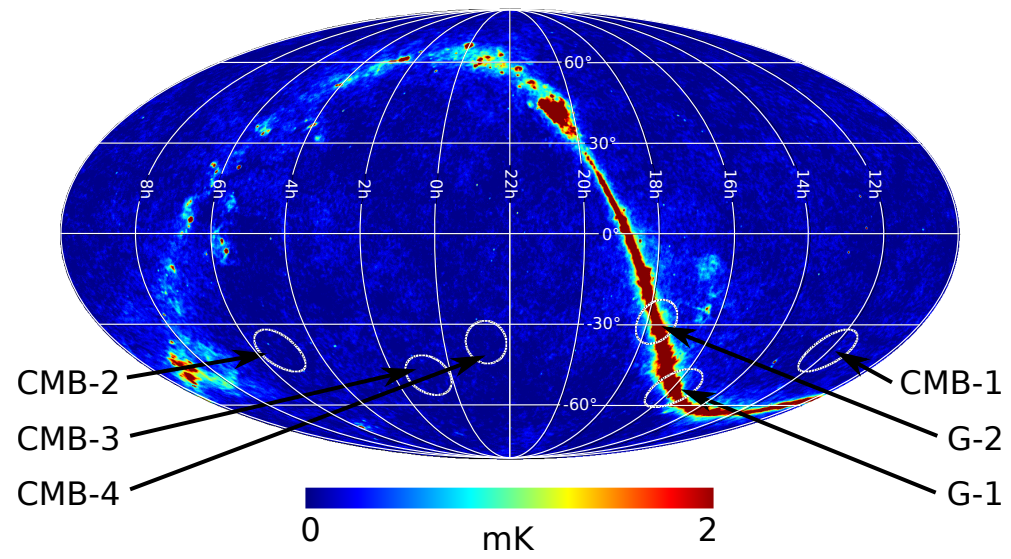

Figure 1: QUIET sky patches (circles) in equatorial coordinates, plotted over the WMAP Q-band temperature map.

\section{Analysis}

Our analysis method converted each-detector time-ordered data (TOD) into CMB polarization power spectra. The analysis flow is as follows. First we calibrated the TOD into units of sky polarization $(\mu K)$. Then we modeled the noise in each CES to understand the statistical uncertainty. We filtered the data in the time domain to remove residual 1/f noise and ground contamination. We rejected data that had too much contamination. After this processing in the time domain, we made maps by projecting the $\mathrm{Q}$ and $\mathrm{U}$ intensities into Galactic coordinates with taking into account 
the telescope pointing information (Fig. 2). Then power-spectrum estimation is performed with pseudo- $C_{l}$ cross correlation technique [5, 6]. To calibrate the analysis pipeline and understand its uncertainty, we performed the same analysis on hundreds of simulations of the entire experiment. We validated our analysis chain with a blind-analysis strategy [7]. QUIET is the first CMB experiment to have adopted a strict blind-analysis policy, in which all data-selection criteria are adjusted and finalized, and the systematic errors are assessed prior to looking at any cosmological power spectrum. This process was described in detail in [2, 8], and have adopted the same policy for both the Q-band and W-band analysis.

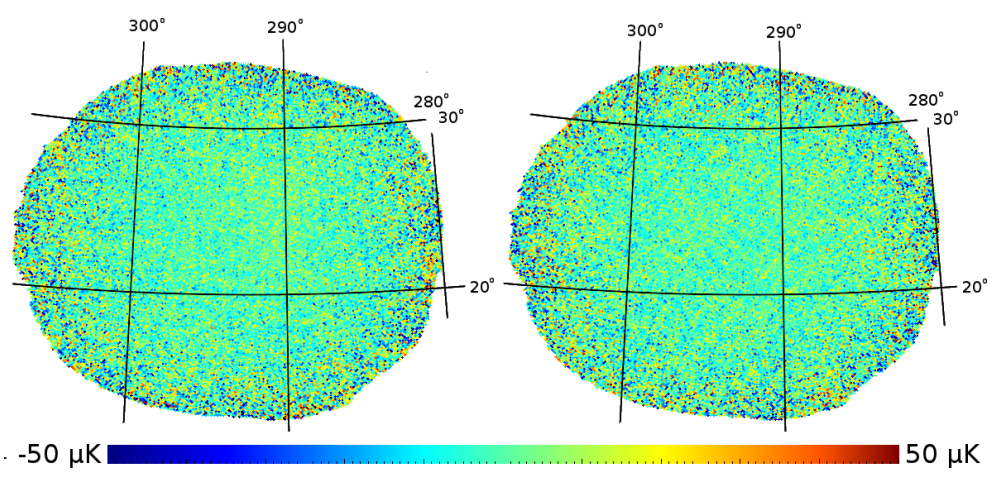

Figure 2: QUIET CMB polarization maps of the CMB-1field in Galactic coordinates at $95 \mathrm{GHz}$. The left (right) panel shows Stokes $\mathrm{Q}(\mathrm{U})$, where the polarization angle is defined with respect to the Galactic North Pole. Note the coherent vertical/horizontal patterns in the Q map, and the diagonal patterns in the U map; these are the expected signature of a pure E-mode signal. No filtering has been applied to this map beyond subtracting the very largest angular scales (ell $>25$ ), to which QUIET is not sensitive.

\section{Results}

The measurements of EE and BB power spectra with Q-band and W-band data are plotted in Figure 3 together with the results by different experiments. The EE spectrum is strongly signaldominated up to $l \sim 800$, and three acoustic peaks are clearly traced, while the BB spectra are consistent with zero within the estimated statistical and systematic uncertainties. The dominant EE power is also visible in the maps shown in Figure 2. Based on these measurements, we constrain the tensor-to-scalar ratio to $r=1.2_{-0.8}^{+0.9}(\mathrm{r}<2.7 \text { at } 95 \% \text { C.L. })^{1}$. This is one of the most stringent upper limit to date.

\subsection{Systematic Errors}

The systematic errors in the power spectra in W-band are shown in Figure 4 The possible contaminations are well below the statistical errors; in particular, the level of spurious B-modes for multipole range relevant for estimation of the tensor-to-scalar ratio, $l \sim 100$, are less than the signal of $r=0.01$. This is the lowest level of BB contamination yet reported by any CMB experiment.

\footnotetext{
${ }^{1} \mathrm{Here}, \mathrm{r}$ is defined as the ratio of the primordial-gravitational-wave amplitude to the curvature-perturbation amplitude at a scale $k_{0}=0.002 \mathrm{Mpc}^{-1}$
} 


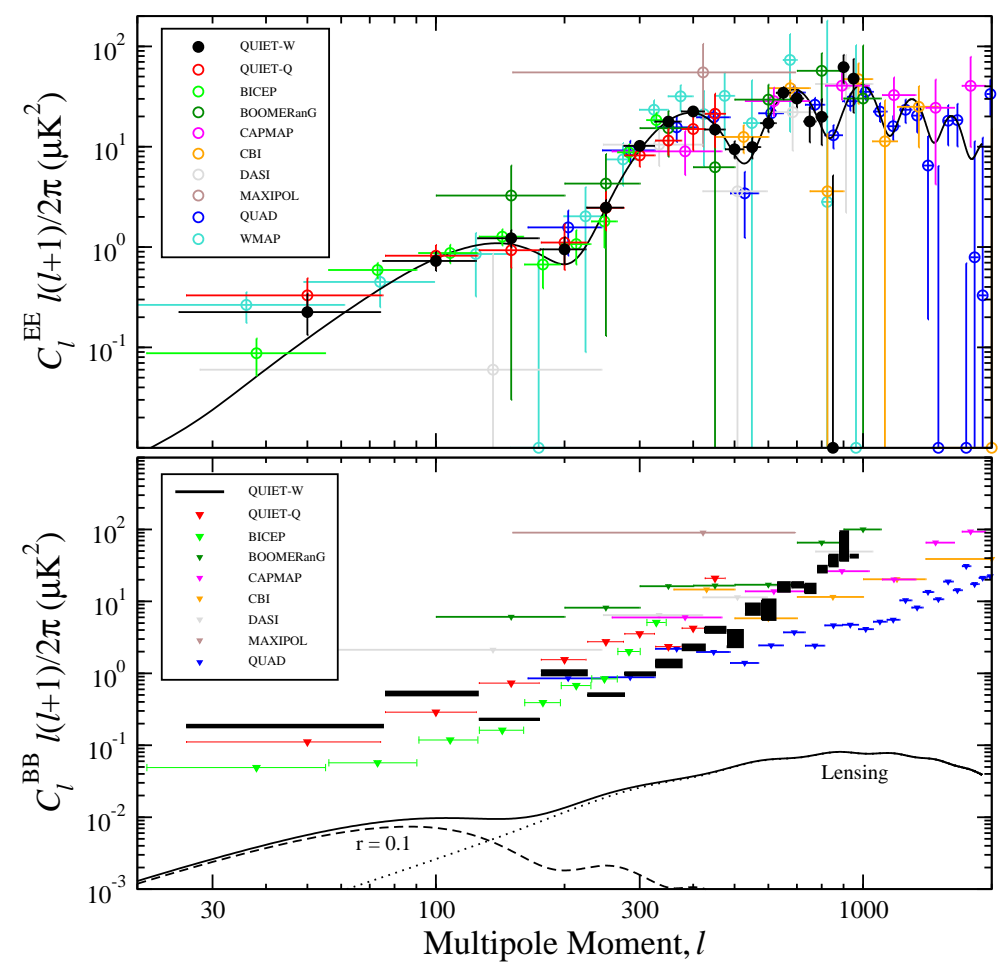

Figure 3: Summary of CMB polarization EE power spectrum (top) and 95\% C.L. upper limits on BB power (bottom) measured by various experiments.

\section{Conclusion}

Motivated by the exciting possibility of detecting the gravitational waves due to inflation, many efforts are underway to develop the instrumentation and methods necessary to search for B-modes. In this proceedings, we have presented the instrument, observation and the latest analysis results from QUIET. The EE spectrum is measured with high $\mathrm{S} / \mathrm{N}$; the BB power spectrum is consistent with zero. The EE spectrum is consistent with a $\Lambda \mathrm{CDM}$ model. The polarization data passed all of the statistical validation tests before looking the power spectrum. A detailed extensive study of potential systematic errors indicate that all systematic effects are below the level of $r \sim 0.01$ at $l \sim 100$. Our observation strategy and analysis frame provide a guide for future experiments in search for the signature inflationary gravitational waves in CMB polarization.

\section{Acknowledgement}

Support for the QUIET instrument and operation comes through the NSF cooperative agreement AST-0506648. Support was also provided by NSF awards PHY-0355328, AST-0448909, PHY-0551142, PHY-0855887, and AST-1010016; KAKENHI 20244041, 20740158, and 21111002; PRODEX C90284; a KIPAC Enterprise grant; and by the Strategic Alliance for the Implementation of New Technologies (SAINT). We are particularly indebted to the engineers and technician who maintained and operated the telescope: Jose Cortes, Cristobal Jara, Freddy Munoz, and Carlos 


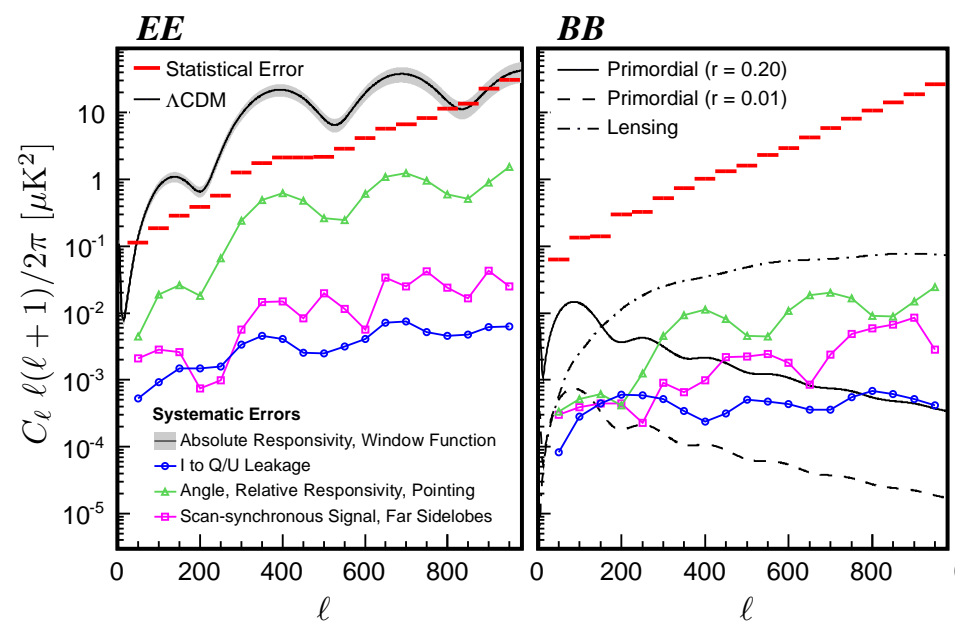

Figure 4: Summary of systematic error assessment for E-modes and B-modes. The red bars indicate the statistical uncertainties in each bin. Blue, green, and purple points correspond to three categories of systematic errors: I-to-Q/U leakage; polarization angles (absolute and relative), relative responsivities and pointing error; and the residual scan-synchronous signals and far sidelobes. The gray band along the $\Lambda$ CDM curve in EE corresponds to the uncertainties of multiplicative factors: absolute responsivity and the window function. For BB, all systematic errors are below the level of $r \sim 0.01$ at $l \sim 100$.

Verdugo. Finally, QUIET was led by Bruce Winstein, who died in 2011 February soon after observations were completed. His intellectual and scientific guidance was crucial to the experiment's success.

\section{References}

[1] M. Zaldarriaga and U. Seljak, All-sky analysis of polarization in the microwave background, Phys. Rev. D55, 1830 (1997)

[2] QUIET Collaboration, First season QUIET observations: Measurements of Cosmic Microwave Background Polarization Power Spectra at $43 \mathrm{GHz}$ in the multipole range $25 \leq l \leq 475$ ApJ. 741, 111 (2011)

[3] Kieran A. Cleary, Coherent polarimeter modules for the QUIET experiment, Proc. SPIE 7741, $77412 \mathrm{~N}(2010)$

[4] O. Tajima el al., Novel Calibration System with Sparse Wires for CMB Polarization Receivers Journal of Low Temperature Physics 167, 936 (2011)

[5] G. Chon et al., Fast estimation of polarization power spectra using correlation functions, MNRAS, bf 350, 914-926 (2004)

[6] F. K. Hansen and K. M. Gorski, Fast cosmic microwave background power spectrum estimation of temperature and polarization with Gabor transforms. MNRAS, 343, 559-584 (2003)

[7] J. R. Klein and A. Roodman, Blind Analysis in Nuclear and Particle Physics. Review of Nuclear and Particle Science, bf 55, 141-163 (2005)

[8] QUIET Collabolation, Second Season QUIET Observations:Measurements of the CMB Polarization Power Spectrum at $95 \mathrm{GHz}$ ApJ. 760, 145 (2012) 